\title{
The Application of Computer Aided Design as a Tool for Building User-Centered Design in Consumer Ceramics' Product Development
}

\author{
Olalere Folasayo Enoch, Universiti Malaysia Kelantan, Malaysia \\ Ab Aziz Bin Shuaib, Universiti Malaysia Kelantan, Malaysia \\ Ramli bin Ismail, Universiti Malaysia Kelantan, Malaysia
}

\begin{abstract}
This paper investigates the knowledge regarding how user-centred design can be built in ceramic consumer products. The paper gives the general overview of ceramics, computer-aided design and its application in ceramic product development. It also illuminates on product emotion, its influence on consumers'behaviour and how it can be integrated into new product conceptualization. Furthermore, the paper analysed the systematic approach in building user-centred design in new product and also reveals how CAD can be used to achieve a user-centred design. In order to test the viability of CAD in achieving user-centred design, a study was performed where a CAD-model of a multi-functional ceramic pot was created and a questionnaire with the image (CAD model) and eight emotions was given to participants so as to know their emotional responses toward the product. The result from the study reveals the viability of computer aided design as tool for building user-centred design in consumer ceramics' product development.
\end{abstract}

Keywords: $\quad$ Ceramics, Computer-AidedDesign(CAD), ConsumerCeramics'Product, ProductDevelopment, Product Emotion, Prototyping, User-Centred Design

\section{INTRODUCTION}

According to Chapman (2009), an approach to sustainable user-centred design reduces the consumption and waste of natural resources

DOI: $10.4018 /$ ijacdt.2012070103 by increasing the resilience of relationships established between consumer and product. Research by Boatwright and Cagan (2010) revealed that people pay for products that address their emotional needs in all types of businesses. Therefore, product emotion is critical to the long-term success of any product that 
customers interact with directly or indirectly. In other words, engaging emotion as a partner to technology will deliver the next market place products that will captivate customers.

Although, collaborations between manufacturers and supplies are becoming increasingly more usual, it is still far from common for the customer to be considered as a fundamental participant in the collaborative design chain (Camarinha-Matos et al., 1999). The different types of customers that will filter the product throughout its life cycle are stakeholders in the process and should be given preferences from the very earliest design stages. Therefore, this paper investigates the knowledge regarding how user-centre design can be built in ceramic consumer products. It reveals how CAD can be used to achieve a user-centred design and also test the viability of CAD in achieving this by performing a study where a CAD-model of a multi-functional ceramic pot was created and a questionnaire with the image (CAD model) and eight emotions was given to participants so as to know their emotional responses toward the product. The result from the study reveals the viability of computer aided design as tool for building user-centred design in consumer ceramics' product development.

\section{CERAMICS OVERVIEW}

Ceramics is the art and science of making useful products for man from inorganic, non-metallic materials by the action of heat and subsequent cooling (CTIOA, 2011). It can also be defined as heat-resistant, non-metallic, inorganic solids that are generally made up of compounds from metallic and non-metallic elements. Although different types of ceramics can have very different properties, in general ceramics are corrosion-resistant and hard, but brittle. Most ceramics are also good insulators and can withstand high temperatures. These properties have led to their use in virtually every aspect of modern life.

The two main categories of ceramics are traditional and advanced ceramics. Traditional ceramics are produced from materials that are obtained from common, naturally occurring raw materials such as clay minerals and quartz sand (Encyclopaedia Britannica, 2012). Traditional ceramics include objects made from clay and cements that have been hardened by heating at high temperatures. These include dishes, crockery, flowerpots and roof and wall tiles. Advanced ceramics includes carbides, oxides, nitrides and many other materials including the mixed oxide ceramics that can act as superconductors. Advanced ceramics requires modern processing techniques, and the development of these techniques has led to advances in medicines and engineering. Figure 1 and Figure 2 illustrate the difference and relationship between traditional and advance ceramics.

The overlapping relationship between traditional and advance ceramics results in the three classifications of ceramic product. These include; artistic ceramics, consumer ceramic products and engineering ceramics. Artistic ceramics are creative ceramic pieces which are aesthetically pleasing; they focus more on the aesthetic values and less on functionality. They are concerned with the beauty, art and the perception of the final output product and the objectives is to create new concept and direction. Some of the factors that are considered in artistic ceramics production are perception, culture and product emotion of the customer. Examples of artistic ceramics include pottery wares, sculptural piece etc. Consumer products in ceramics are ceramic products produced meanly for personal or household use. The products focus on both the functionality and aesthetic values and the objectives are to solve problems, meet need and also create new innovations. The product includes some house hold utensils and fittings such as sanitary wares, kitchen wares, table wares, etc. Engineering ceramics is the science and technology of creating objects from inorganic, non-metallic materials. This is done either by the action of heat, or at lower temperature using precipitation reactions from high purity chemical solutions. The term includes the purification of raw materials, the study and production of the chemi- 
11 more pages are available in the full version of this document, which may be purchased using the "Add to Cart" button on the product's webpage:

www.igi-global.com/article/application-computer-aideddesign-tool/70393?camid=4v1

\section{Related Content}

Learning 3D Face Deformation Model: Methods and Applications

Zhen Wen, Pengyu Hong, Jilin Tu and Thomas S. Huang (2004). 3D Modeling and

Animation: Synthesis and Analysis Techniques for the Human Body (pp. 317-340).

www.igi-global.com/chapter/learning-face-deformation-

model/4174?camid=4v1a

In Memorial and Serenity: Introducing VR Grave Mourning in the Chapel of St. Michael in Macao

Ka Man Ng and Carlos Sena Caires (2022). International Journal of Creative Interfaces and Computer Graphics (pp. 1-17).

www.igi-global.com/article/in-memorial-and-serenity/308300?camid=4v1a

Shameless Selfie-Promotion: Narcissism and Its Association With Selfie-

Posting Behavior

Eric B. Weiser (2018). Selfies as a Mode of Social Media and Work Space Research

(pp. 1-27).

www.igi-global.com/chapter/shameless-selfie-

promotion/191369?camid=4v1a

A Simple Physically-Based 3D Liquids Surface Tracking Algorithm

Gonçalo N. P. Amador and Abel J. P. Gomes (2012). Innovative Design and Creation of Visual Interfaces: Advancements and Trends (pp. 306-317).

www.igi-global.com/chapter/simple-physically-based-liquids-

surface/64060?camid=4v1a 